\title{
Pensar la sociedad a través de la educación
}

\section{Viewing society through education}

DOI: https://doi.org/10.32870/dse.v0i23.928

\section{Eduardo Solano Vázquez*}

\begin{abstract}
Resumen
En este texto se ha pensado la sociedad moderna-contemporánea, para lo cual se acudió al asunto de la educación. En otras palabras, el escrito hace una reflexión social y política del presente a través de la temática educativa. Por otro lado, el artículo se sostiene en una perspectiva interdisciplinar en tanto que confluyen saberes filosóficos, jurídicos, antropológicos y sociales, los cuales se encargan de analizar la sociedad y la educación. Asimismo, lo que se ha escrito busca contribuir en los estudios que señalan el factor político de la educación, de ahí que el saber se relaciona con el poder, puesto que los que generan y significan el espacio-tiempo también lo dirigen.
\end{abstract}

Palabras clave: educación - escuela - sociedad - política - dominación.

\section{Abstract}

This text reflects upon modern-contemporary society, as seen through the issue of education. The article follows an interdisciplinary perspective in which philosophical, legal, anthropological, social knowledge converges to analyze society and education. Likewise, it seeks to contribute to the studies that point to the political factor of education in which knowledge is related to power, since those who generate and signify space-time also direct it.

Key words: education - school - society - politics - domination.

Las luces, que han descubierto las libertades, inventaron también las disciplinas. Michel Foucault. Vigilar y castigar. Nacimiento de la prisión

Para Mariela Alejandra Muñoz

\section{Introducción}

El propósito de este texto es pensar la sociedad a través de la educación. Por ello, en el primer apartado se ha establecido la relación entre educación escolar y sociedad, a partir de ahí se reflexiona respecto a las implicaciones que la educación tiene en el presente histórico de una

* Doctor en Estudios Latinoamericanos. Líneas de investigación: filosofía e historia de las ideas: cultura, política, educación. Universidad de Guadalajara, México. pumalibro@hotmail.com 
sociedad. Es preciso decir lo siguiente, la sociedad que aquí se considera es la moderna-contemporánea; esta cuenta con un Estado-nación que necesita establecer criterios de sociabilidad para que su población no se disgregue in extremis, ya que, sin un criterio y valores para la población, el Estado-nación es lógica y fácticamente imposible.

El segundo apartado aborda de manera específica la conformación de la sociabilidad y, para que ella se objetive, requiere la enseñanza de los valores hegemónicos, además, es imprescindible que también sean practicados. Así pues, la educación escolar realiza tareas en aras de introducir y enseñar al educando a vivir en sociedad, baste como ejemplo lo siguiente: en el contexto contemporáneo, lo que se quiere alcanzar es la libertad de competencia, y por ello, hoy se le enseña al educando a ser competente en cada una de las tareas solicitadas por los docentes, para que al incorporarse al mercado de trabajo haga sus actividades con eficiencia.

El tercer apartado hace énfasis en la dimensión sociopolítica de la educación, pero para pensar en torno a la posibilidad de una transformación en aras de conseguir la igualdad y la justicia en las relaciones sociales, económicas y políticas. Así pues, se abandona el "pesimismo" que subyace en los dos primeros apartados, y se vislumbran otros escenarios sociopolíticos. También se menciona que el educando es de suyo imperfecto, pero ello no cancela su capacidad de aprendizaje, este lo hace mejorar cotidianamente en sus actividades en tanto que las puede corregir y resignificar.

En la conclusión se insiste en la imposibilidad de la neutralidad en la educación. Sin embargo, no se renuncia a ella puesto que se vislumbra la posibilidad de modificar los objetivos y los contenidos en la enseñanza-aprendizaje; con ello es factible que se le dé otro sentido a la realidad del educando y la sociedad. También se recalca que el estudio y la comprensión en torno a la educación escolar requieren una perspectiva política, y no solamente didáctica.

\section{Educación y sociedad}

La educación escolar no solo se dedica a la enseñanza y aprendizaje de asuntos intelectuales y técnicos, también tiene injerencia en los comportamientos y las acciones sociopolíticas. Asimismo, los Estados educan a su población para introducirla de manera gradual y paulatina en las leyes y las costumbres, fundamentales para la organización y el funcionamiento del espacio público. En lo que respecta al contexto mexicano, María Espinosa apunta:

El siglo XX en México, en el terreno educativo, comienza y termina discutiendo, entre otros temas: la federalización educativa, la educación nacional e integral; la necesidad de la educación indígena y rural; la diferencia entre instrucción y educación y la imperiosa necesidad de la enseñanza moral [...] El siglo XX mexicano inicia con una escuela primaria donde domina el trabajo, el silencio y el orden, donde se pretende desarrollar una educación integral y en la que se ha distinguido claramente la diferencia entre educar e instruir, asumiendo la escuela pública como tarea central la de educar (Espinosa, 2020: 1).

Diólo pos sobre Educación año 12 | número 23 | julio-diciembre 2021 | ISSN 2007-2171 
La escuela tiene una independencia relativa respecto al Estado, eso le permite presentarse como una institución neutral. Sin embargo, su funcionamiento se encuentra imbricado con la estructura general del Estado. Así pues, en su condición de institución del Estado, se convierte en un factor fundamental para inculcar la sociabilidad a los ciudadanos.

La educación escolar es una práctica racional; su racionalidad consiste en el uso de las metodologías y las didácticas, y con ellas se establece la transmisión de los saberes. Asimismo, no se puede omitir que su racionalidad también reside en su estatus de institución del Estado. Por ello es que la escuela educa de acuerdo a las necesidades del Estado y no de lo que requieren los profesores y los alumnos en lo particular. Sin embargo, los maestros no son meros transmisores y receptores de los saberes enseñados y aprendidos en la escuela.

La educación escolar adquiere una doble función dentro del Estado: por un lado, transmite el modus vivendi socialmente hegemónico, pero también se encarga de generar el capital humano para el desarrollo económico. En otras palabras, el objetivo de la educación escolar moderna-contemporánea es generar ciudadanos que acaten las leyes-costumbres y, a su vez, que también sean productivos-útiles respecto al modo de producción. Aunque no siempre se logra el objetivo a cabalidad, lo relevante es que la educación escolar no busca formar para un más allá, sino para el presente de las sociedades, tanto en su deriva política como económica. Al respecto, Manuel de Puellez señala:

El Estado liberal del siglo XIX y buena parte del XX hará suya la idea de la educación como factor de integración política y de control social. Desde el punto de vista de la integración política, el Estado liberal concebirá la educación como elemento sustancial para el logro de una nueva lealtad y procurará que las clases medias y superiores, base del nuevo régimen representativo, tengan fácil acceso a la educación secundaria y superior (aunque ambos tipos de enseñanza suministrarán los nuevos cuadros que la nueva administración necesita, la integración política seguirá siendo uno de los objetivos principales) (De Puellez, 1993: 45).

La escuela, como institución del Estado, tiende a provocar la implosión de los conflictos sociales en tanto que se jacta de ser neutral. Sin embargo, ella amalgama y esparce el modus vivendi socialmente hegemónico. Ahora bien, en la educación escolarizada están depositadas las expectativas de la sociedad, pero en esta hay grupos que tienen menor o mayor injerencia; siendo más precisos, unos pueden ejercer el poder a través de las instituciones y otros no. Es el poder ejercido mediante la institución el que estipula el cómo se vive en sociedad.

La educación escolarizada conforma al ser humano en su dimensión individual y social. Es decir, ella construye la subjetividad y la manera en que se percibe el cuerpo en el ámbito social e histórico. La conformación de la subjetividad es cardinal para el establecimiento y despliegue de una relación social, por ejemplo, una mentalidad medrosa va a influir en la concepción y la experiencia de un cuerpo sumiso y propenso a ser explotado, dirigido. 
La importancia que la educación escolar tiene en la conformación del espacio social e histórico es insoslayable, y permite decir que la hegemonía sociopolítica no es una acción esporádica ni deudora de la ocurrencia; tampoco tiene que ver con los instintos o la fuerza física. Por el contrario, la hegemonía sociopolítica es un proyecto, el cual tiene una base racional que se va desplegando en distintas instituciones, aunque la más idónea para ese cometido es la escuela. Cabe señalar que no se asume un determinismo social, pero no se puede eludir el factor hegemónico en las relaciones sociopolíticas.

La educación escolar conforma la sociabilidad históricamente requerida. Por otro lado, la sociedad requiere un modo de organizarse, cuya organización es imposible sin unas reglas de convivencia. Por ello es importante señalar lo siguiente: no hay sociedad que se desarrolle mediante la ocurrencia. Así pues, toda sociedad, por minúsculo que sea su nivel de desarrollo civilizatorio, se sostiene en un régimen de valores.

\section{Educación y enseñanza de los valores hegemónicos}

La adscripción a la ciudadanía de un Estado permite la obtención de derechos y obligaciones, que permiten a los hombres y las mujeres tener óptimas condiciones en el espacio público. Sin embargo, en nombre del bien público y de la nación, también se transgrede y pulveriza la dignidad y se cometen actos deleznables. Así pues, pertenecer a un Estado no significa que de facto estén cubiertas las necesidades, y mucho menos que estén protegidas las aspiraciones sociopolíticas. Respecto al contexto mexicano, José Narro Céspedes comenta:

La Constitución de los Derechos de los Pueblos Indígenas nunca fue incorporada a la Carta Magna mexicana. Los gobernantes lo impidieron con todos sus medios. Pero el documento existe y es vigente [...]. El no cumplimiento de estos acuerdos ha configurado escenarios de pobreza y confrontación, al no existir las herramientas jurídicas para garantizar sus derechos. La apuesta de las cúpulas políticas y económicas de reducir la compleja problemática indígena a un asunto solo de programas sociales ha fracasado (Narro, 2018: 1).

Los derechos y las obligaciones son medulares para organizar el espacio social, pero se requieren otros elementos para perpetuarlo, de ahí que el sistema escolar tiene un rol importante en cuanto a la organización de la sociedad. Los modelos didácticos contienen formas de organización social, por ejemplo, el modelo tradicional de enseñanza expresa una relación social rígida, en la que la última palabra la pronunciaba el docente, el padre, el Estado. Ahora bien, las didácticas actuales que celebran por doquier la creatividad y el ingenio de los niños se parecen demasiado a la forma en que se llevan a cabo las relaciones sociales entre adultos (las cuales van desde las laborales hasta las comerciales), donde se celebra la flexibilidad y la iniciativa; lo anterior solo es una variante de la descentralización del poder, misma que esparce la idea de 
que la sociedad contemporánea es preponderantemente libre, por lo tanto, el individuo puede hacer lo que le plazca.

La escuela es cardinal en cuanto a la conformación de la estructura sociopolítica del Estado. Por ello, el señalamiento de algunos valores diseminados en el sistema escolar contemporáneo sirve para comprender la manera en que se gesta la hegemonía. Es imprescindible indicar que la escuela produce y reproduce los comportamientos de la hegemonía:

\begin{abstract}
Todo sistema de enseñanza institucionalizado (SE) debe las características específicas de su estructura y de su funcionamiento al hecho de que le es necesario producir y reproducir, por los medios propios de la institución, las condiciones institucionales cuya existencia y persistencia (autoreproducción de la institución) son necesarias tanto para el ejercicio de su función propia de inculcación como para la realización de su función de reproducción de una arbitrariedad cultural de la que no es el productor (reproducción cultural) y cuya reproducción contribuye a la reproducción de las relaciones entre los grupos o las clases (reproducción social) (Bourdieu, Passeron, 1972: 95).
\end{abstract}

Hacia finales del siglo XX y principios del XXI, en México se propuso el modelo de "educación por competencias"; este no solo es para fomentar y desarrollar el capital humano, también esparce y afianza un modus vivendi socialmente hegemónico, al cual podría pensarse como un ethos; es decir, a través de la educación por competencias se transmiten e inculcan los valores requeridos para vivir en sociedad, más allá de que esté en boga la narrativa de la ausencia de valores. Sin embargo, ninguna sociedad carece de ellos, solo que estos no siempre son compartidos, y así, el consenso solamente funge de supuesto. En lo que se refiere al contexto contemporáneo, lo que impera son los valores empresariales:

La educación por competencias nace desde el seno del sector empresarial y es impulsada por los organismos que regentan el sistema capitalista internacional [...] Para las empresas y para las "competencias", los valores y actitudes que desean promover están en función del sometimiento dócil a la voluntad del empresariado. En ese esquema la capacidad "crítica", es decir, reflexiva y comprensiva, no interesa. Y, aunque se habla de promover el "diálogo" se condena la lucha cívica por los derechos, de la cual el estudiantado de todas las generaciones ha aportado a la sociedad (Beluche, 2016: 1).

El modelo de educación por competencias se sostiene -y fomenta- en el ideal de un hombre-sociedad proclive a la ganancia, esta se va a suscitar mediante la inteligencia: “El capital no significa sólo la concentración del dinero, sino también del agua en las represas, del mineral bajo la tierra, de la inteligencia en un banco de ingeniería alejado de quienes ejecutan" (Serres 2013: 67). Así pues, ya no se obtienen los beneficios de manera feroz o cuasi salvaje, la sociedad moderna-contemporánea no tiene permitido ser bárbara, por lo menos en cuanto a la forma, no puede darse ese desliz. Asimismo, si el objetivo principal es ganar, es necesario que se esta- 
blezcan los medios adecuados para que se dé con eficacia. Así pues, los cursos de actualización -y hasta los de superación personal- son solicitados para poder estar capacitados en un mundo que exige y demanda ser eficiente; también se supone que ahora ya no hay explotación, en tanto que la empresa-fábrica ofrece la oportunidad de crecer, por lo tanto, todo depende de la disposición y la inteligencia. En este sentido, la aspiración por el bienestar y la abundancia no desaparece en una sociedad que se jacta de ser racional y que tiene bajo control sus modos de producción económica y social.

La sociedad moderna-contemporánea se sostiene en los ideales de libertad, igualdad y competencia, sin embargo, esta no garantiza que la ganancia sea para todos. Asimismo, nadie prohibe ser libre y tampoco competir en el mercado, pero hay que tener en cuenta que los demás también tienen ese derecho. Ahora bien, los que se benefician son los que estén más capacitados, tú no puedes quejarte ni decir que tu derrota es injusta o que el sistema te excluye, ya que no podrás negar que las oportunidades de libertad, igualdad y competencia son democráticas. Así pues, si no te va bien, es porque no eres capaz de conducir tu libertad y de hacer con pericia las cosas. La derrota cae exclusivamente en el individuo, mientras que la sociedad sigue funcionando, pasando desapercibido que hombres y mujeres se deprimen, acuden al psicólogo o a los cursos que ofertan la felicidad y el manual para obtener ganancias en cualquier actividad.

La sociedad moderna-contemporánea esparce la idea de que todo es posible, pues ella promueve y detona la libertad. Además, establece las condiciones para la realización de la competencia. Sin embargo, no todo es posible y las situaciones no suceden solo con decretarlas, esa manera de pensar no hace más que ridiculizar y disminuir la potencia e importancia de la acción, con esta se puede comenzar a virar cualitativamente el ámbito sociopolítico, con el propósito de que la desigualdad no sea el principio y fin de la existencia.

Los derechos y valores ofrecidos por la sociedad hegemónica son indispensables para mantener el estatus quo y el orden, pero carecen de un impacto real en cuanto a mejorar las condiciones materiales de vida en la población. Por ejemplo, la libertad de competir oculta la dominación de la que son objeto hombres y mujeres, la ocultación se da a través del afianzamiento de la siguiente creencia: las actividades que uno se propone no tienen otro detonante más que la voluntad y los deseos personales, por lo que nadie influye en lo que se realiza, pero eso no es así, ya que en la medida que uno se encuentra dentro de la lógica y las prácticas hegemónicas, las actividades que uno se propone resolver tendrán que ser útiles para la sociedad; de lo contrario, carecerán de valor en tanto que no reportarán ninguna ganancia para la sociedad y su modo de producción. Así pues, bajo el mito de la competencia se mantienen y desarroIlan las estructuras del modus vivendi socialmente hegemónico. En lo que respecta al contexto colombiano, Juan Gutiérrez señala que el Estado potencia y protege a la empresa y la libertad económica: 
La empresa, como base del desarrollo, tiene una función social que implica obligaciones. El Estado fortalecerá las organizaciones solidarias y estimulará el desarrollo empresarial [...] La ley delimitará el alcance de la libertad económica cuando así lo exijan el interés social, el ambiente y el patrimonio cultural de la Nación (Gutiérrez, 2013: 1).

El Estado moderno se ha adaptado a las situaciones históricas, lo cual ha propiciado formas distintas de ejercer el poder. Es decir, si en un principio fue pertinente un poder cuasi absoluto, en la actualidad es imprescindible un poder flexible que hace suponer lo siguiente: la sociedad contemporánea es incluyente y pacifista. Sin embargo, la flexibilidad del poder no se ha traducido en una mejoría constatable en cuanto a la disminución de la desigualdad social, jurídica y económica. Por otro lado, la educación escolar también está en concordancia con la flexibilidad del poder, basta como ejemplo que a los alumnos de nivel básico se les apruebe porque sí, lo cual genera una nivelación escolar perniciosa, y sus repercusiones se verán a mediano y largo plazo. En el contexto mexicano se suscita lo siguiente:

Se eliminará la boleta de calificaciones que registra las evaluaciones mensuales y las calificaciones al final de cada año escolar; será sustituida por una Cartilla de la Educación Básica, en la cual se consignarán las evaluaciones de los menores, pero éstas se realizarán por periodos de tres años [...] De igual forma, se eliminará el concepto de aprobado y reprobado, para sustituirlo por el concepto de "promovido al siguiente grado (Robles, 2011:1).

En las sociedades no hay ausencia de poder; que este hoy en día ya no opere de manera cuasi absoluta y se muestre flexible, es dable por las condiciones históricas y económicas. Asimismo, que las opiniones de cada uno puedan ser exhibidas en las redes sociales y también se cuente con una variedad de opciones televisivas, de partidos políticos, causas sociales a raudales y consumo de mercancías al por mayor, no significa que el poder de decisión respecto a la estructura sociopolítica sea de facto democrático, este sigue siendo exclusivo de una minoría, la cual detenta el poder económico, pero requiere sostenerse en el poder político y en el científico-cultural, sin los cuales le sería imposible establecer la hegemonía. Así pues, la elite económica no ejerce directamente el poder político y tampoco el científico-cultural; se puede decir que lo delega en los políticos de profesión y los expertos en la materia.

El poder se ejerce, de no hacerlo, se convierte en trivial-superfluo. Ahora bien, en la propuesta de un canon de vida, ya hay indicios del ejercicio del poder puesto que a partir de ahí se regulan y realizan las conductas, y así, los que no se incorporen y practiquen el canon carecerán de relevancia social (ejercicio simbólico del poder), lo que trae como consecuencia el aislamiento y la pérdida de injerencia en la sociedad: economía, educación y política. Por otro lado, el modelo social hegemónico no se imita de inmediato, ni es suficiente con que la familia y los medios de comunicación lo transmitan; por lo tanto, para que el modelo social hegemónico se afiance 
y perdure tiene que inculcarse de manera sistemática; aquí es donde la escuela se encarga de la sistematización y enseñanza del modus vivendi socialmente hegemónico:

Si la relación constitutiva de la escuela es la interacción docente-alumno, en ella el poder es privativo del maestro, dada la asimetría que surge del hecho de que los agentes movilizan recursos diferenciales en sus interacciones. Desde luego, el poder del docente no sólo se vincula con los recursos del conocimiento que puede movilizar en dicha interacción sino también, y como se adelantará, con el hecho de que exhibe un mandato de la sociedad para conducirla (Betallán, 2003: 692).

La educación escolar no es neutral, ya que educa en y para el funcionamiento de la sociedad. Ahora bien, la supuesta neutralidad de la escuela propicia que no se considere su carácter político. Sin embargo, la prioridad de la educación escolar, al ser una institución del Estado, es formar al estudiante para que ejerza de la mejor manera posible sus roles macrosociales (ciudadano, trabajador, consumidor), lo cual no impide que este tipo de educación afecte la dimensión microsocial del alumno y presente diferencias tangibles respecto a su familia y amigos, en lo que respecta al rubro salarial y también al de la asimilación de los códigos culturales socialmente aceptables. No obstante, es importante expresar que ese es un aspecto secundario o de segundo rango en tanto propósito formativo de la educación escolar, ya que el objetivo medular es atender las necesidades macrosociales, puesto que ellas rigen y soportan el espacio social y, por supuesto, el proyecto cultural-civilizatorio hegemónico.

Una de las características de la sociedad moderna-contemporánea es que funciona en términos sistémicos y organizacionales. Esta manera en la que se realiza la sociedad permite el control de la subjetividad-corporalidad de los individuos, lo cual se ve objetivado en las predisposiciones respecto a ciertas elecciones sociopolíticas, mismas que aparentemente se toman haciendo uso de la libertad y ejerciendo el derecho a ser diferentes. Sin embargo, las decisiones respecto a la libertad y a la diferencia se encuentran condicionadas por el poder político, y también por el poder científico-cultural.

\section{Educación y posibilidad de cambio sociopolítico}

Si se parte del hecho de que la sociedad hegemónica tiene las situaciones y las cosas bajo control, entonces ¿por qué hay posturas y proyectos sociopolíticos que insisten en mostrar sus insuficiencias? ¿cuáles son los objetivos de las acciones sociopolíticas que quieren modificar el estado de cosas existente? Que la sociedad hegemónica controle los comportamientos y las expectativas sociopolíticas no significa que las acciones para destruir las estructuras del proyecto cultural-civilizatorio dominante sean infructuosas. En este sentido, algo que es primordial criticar y superar en la sociedad contemporánea es el modelo de educación por competencias, y no porque sea preferible la mediocridad en torno al aprendizaje y el saber, sino porque la propuesta de educación por competencias es parcial e insuficiente, en tanto que únicamente

Diólo os

sobre Educación año 12 | número 23 | julio-diciembre 2021 | ISSN 2007-2171 
responde a la lógica y práctica empresarial. Ahora bien, la disputa por el qué y el cómo se enseña en la escuela, no es algo meramente instrumental. Es decir, no solamente es por el tipo de didáctica que ha de establecerse en la escuela, sino que es ante todo una disputa política, en el sentido de que lo enseñado en la escuela condiciona la existencia de hombres y mujeres, y también la de los grupos sociales y étnicos a los que pertenecen:

Las Pedagogías Alternativas brindan a cada educando una táctica, un orden y un estilo educativo que le sea propio y al facilitador le permite encontrar un equilibrio entre conocimiento y potencialidades del participante, para hacer de éste un ser más autónomo en su desarrollo intelectual, brindándole desde su realidad las posibilidades de construir un camino hacia un futuro deseable [...] hablar de Pedagogías Alternativas se refiere a la búsqueda de una enseñanza de la emancipación, con base en la práctica de la acción transformadora que rechace la idea de neutralidad, de dependencia cognitiva, de homogeneidad y de pasividad. Es entender la educación como vía de cambio, como medio de construcción de la ciudadanía para adaptar y transformar la realidad (Alirio et al., 2016: 239-240).

La hegemonía sitúa su lógica y sus prácticas en la defensa y perpetuación del estatus quo y lo correcto. Sin embargo, la corrección sociopolítica es insuficiente para la construcción de una sociedad equitativa y plural. En este sentido, es oportuno el deseo y la acción para lograr cosas distintas en el espacio histórico, social y político. Por otro lado, es una verdad de Perogrullo que las decisiones cruciales se desenvuelven en la incertidumbre y no se puedan controlar, ni tampoco predecir lo que sucederá con ellas; a lo sumo se previene, pero aún con la prevención, la decisión que se haya tomado puede ser que a largo plazo no satisfaga. Así pues, es razonable asumir que ninguna decisión es perfecta, pero de alguna manera permite perfilar la modificación del estado de cosas. Ahora bien, es oportuno preguntar ¿cuáles son las alternativas respecto al proyecto sociopolítico que cree fervorosamente en la libertad y la competencia en el mercado?

La imperfección es la condición del ser humano en tanto finito, y eso incide en sus acciones, sus creaciones. Es decir, por más esfuerzos en pos de alcanzar la perfección, esta solamente es un modelo-guía, por lo tanto, exigir relaciones sociales perfectas es olvidar lo imperfecto del ser humano. Sin embargo, también es pernicioso reivindicar la resignación y suscribir que "lo que ocurre en el mundo es inevitable". Así pues, el conformismo no es la opción, si lo que se quiere es soterrar las relaciones de desigualdad e injusticia. Ahora bien, recordar lo imperfecto del ser humano y, por ende, de las relaciones sociales que realiza, es con el propósito de no esperar panaceas ni situaciones armónicas. Asimismo, pensar desde lo imperfecto en cuanto a la acción humana y las relaciones sociales que ella establece, permite vislumbrar otras realidades históricas y sociales distintas a la hegemónica: 
La historia del hombre es una historia imperfecta: la perfección en este sentido, es un atributo divino. Más aún: si el mundo, o el hombre, fuesen perfectos, para qué existiría un sentido de reparación [...] El mundo es irreparable. Mientras lo habitemos así será, y ello es lo que nos permite crear, o apreciar la belleza [...] Debemos comprender la historia como las grietas que se abren en el mundo-naturaleza: allí el hombre irrumpe en el espacio-tiempo (Taub, 2020: 1).

Las relaciones sociales, en tanto que son precedidas por la acción humana, son imperfectas, pero eso no es el acabose pues ellas pueden ser mejoradas, sobre todo, cuando no logran satisfacer las necesidades básicas de la población: vivienda, salud, trabajo. En este sentido, el modus vivendi socialmente hegemónico, el cual organiza el espacio social y también estipula los parámetros de libertad y competencia, no ha logrado satisfacer a cabalidad las necesidades; de ahí que no es un absurdo proponer otros proyectos culturales-civilizatorios:

Conocíamos el capitalismo salvaje, pero ahora también hay un capitalismo responsable, sostenible, con rostro humano, pero todos sabemos lo que ha hecho [...] No podemos hacer cooperativas si no hay personas dispuestas a perder, que su objetivo no sea solamente buscar una alternativa de producir y vivir, sino que estén en la empresa sabiendo que podrían estar mejor en otro sitio, porque es una forma de combatir [...] Si hacemos productos detergentes que no caigan en la generación de necesidades absurdas, creemos que estamos contribuyendo a un cambio en el estilo de vida, a un cambio en la lógica de la producción, que se limita a lo necesario (Del Pozo, 2016).

La flexibilidad del poder que rige en el contexto actual puede dar cabida a las alternativas en el espacio social hegemónico. Sin embargo, no lo hace para potenciarlas sino para controlarlas (implosión del conflicto social). Asimismo, los Estados y los grupos sociales hegemónicos consideran políticamente incorrecto y antidemocrático expresar su régimen de dominio, considerando que la retórica de la libertad y la inclusión de las diferencias son el ideal y el estandarte de la sociedad global:

Cuando visité la provincia de Yen Bai en los montes al norte de Vietnam en agosto, conocí a una mujer que ahora tiene electricidad para ayudar a moler arroz, bombear agua, hacer funcionar ventiladores y alumbrar su vivienda de una habitación para que sus hijos puedan estudiar por la noche, gracias a un proyecto de electrificación financiado por el Banco Mundial en ese país [...] Cuando se les da la oportunidad y dondequiera que estén, las personas desean construir una vida mejor para ellas mismas y para sus hijos. En terreno fértil, ese impulso puede contribuir al establecimiento de una sociedad global saludable y próspera (Zoellick, 2007).

La escuela, mediante lo que enseña, sostiene el modus vivendi socialmente hegemónico. Es decir, la impartición de saberes teóricos y prácticos de raigambre liberal y moderna contribuyen 
al establecimiento y acción de la hegemonía, pues se estipula un tipo exclusivo de conocimiento, saber y aprendizaje, a través del cual se evalúan los méritos y deficiencias de los alumnos: "La inflexibilidad del cientificismo es parte de la mezcla que hay que retirar. Debemos reconocer que, además de apoyarse en el conocimiento de las causas eficientes, las elecciones científicas están cargadas de valores y propósitos" (Wallerstein, 2005: 21). La escuela contribuye a la hegemonía, pero su contribución está velada en tanto que pregona la búsqueda del bien y el desarrollo del hombre-sociedad.

La pretensión por virar el mundo repleto de capitalismo hasta en sus protestas y saberes, requiere de la educación en tanto práctica de libertad, en el sentido de que ella puede abordar las subjetividades y corporalidades en pos de provocar la imaginación y coadyuvar en la realización de otros proyectos culturales y civilizatorios. Es cierto, con la educación se establece la hegemonía, pero también mediante ella se puede derruir, ya que tiene la facultad de conformar el pensamiento y la acción. Así pues, sí es posible deconstruir el pensamiento que solo cree en la libertad y competencia de raigambre liberal:

Hace falta abrir un tiempo y un espacio de desorientación epistémica y ontológica entre, por un lado, la aprehensión cognitiva y emocional de aquellos "cuyas diferencias escapan a nuestras pretensiones de negarlos, cambiarlos, asimilarlos, degradarlos [...] Propongo aquí una pedagogía que reconoce las fuerzas psíquicas y afectivas: se trata de trabajar con los procesos de resistencia no lineales inherentes a toda enseñanza-aprendizaje [...] El poder se encastra cuando las evidencias anecdóticas se ponen en circulación y asumen a priori el valor de certezas manifiestas y necesarias dentro de comunidades privilegiadas aisladas (Taylor, 2014: 1).

La educación ha sido toral para los proyectos culturales-civilizatorios. Así pues, no hay reforma, mantenimiento del estatus quo o transformación histórica, social y política que no recurra a la educación. Por otro lado, es conveniente pensar y poner en práctica un proyecto educativo que detone prácticas de libertad, pero que no exagere en sus expectativas, por ello se debe tener en cuenta que la educación es para alguien históricamente situado.

\section{Conclusión}

La educación escolar forma en dos sentidos, ambos no se contraponen, sino que se complementan. En otras palabras, lo que se enseña a nivel teórico y práctico en la escuela es idóneo para conformar y sostener el modus vivendi socialmente hegemónico. En este sentido, la educación es siempre intencional y la neutralidad no es más que la retórica, esta coadyuva a la despolitización del pensamiento y la práctica educativa. Ahora bien, la despolitización de la educación escolar no significa que la escuela deje de realizar tareas en pos del funcionamiento y consolidación de un proyecto cultural-civilizatorio en específico. 
La comprensión de la sociedad y del proyecto cultural-civilizatorio a través de la educación requiere una perspectiva política respecto al análisis de la educación escolar, por lo tanto, es oportuno observar lo que ella enseña y lo que no, y ver la repercusión de lo enseñado en el exterior de las aulas. Es decir, se debe dar cuenta de la jerarquía y repercusión sociopolítica de los saberes inculcados, ello implica leer la didáctica más allá de lo instrumental o los procedimientos que facilitan el aprendizaje.

La educación siempre pretende perfeccionar al ser humano. Sin embargo, con ello pierde la referencia de la condición imperfecta de aquel y lo lleva al límite de sus capacidades, lo que propicia la desesperación por no tener las situaciones bajo control. Por otro lado, el ser humano no está determinado de una vez y para siempre, y así, mediante la imperfección que lo constituye, es que le es posible proponerse aprender, experimentar; en otras palabras, educarse en sentido lato.

La educación también puede contribuir a minar el estatus quo. Es decir, los proyectos de educación con perspectiva libertaria y en aras de la igualdad y la justicia no son imposibles de realizar en la historia. Sin embargo, es deseable que asuman como principio que el educando es de suyo imperfecto, así no se le exige y tampoco se le promete demasiado. Tal vez es momento de pensar y practicar una educación ajena a las panaceas. Por otro lado, cabe recordar que la educación por competencias también es una panacea, por más ciencia, administración y tecnología que contenga su práctica y narrativa.

\section{Referencias bibliográficas}

Bourdieu, P.; J. Passeron (1972). La reproducción. Elementos para una teoría del sistema de enseñanza. Barcelona: LAIA

Serres, M. (2013). Pulgarcita. El mundo cambió tanto que los jóvenes deben reinventar todo: una manera de vivir juntos, una manera de ser y de conocer. México: Fondo de Cultura Económica.

Wallerstein, I. (2005). Las incertidumbres del saber. Barcelona: Gedisa.

\section{Referencias electrónicas}

Alirio, Á.; B. Africano; M. Febres-Cordero; T. Carrillo (2016). Una aproximación a las pedagogías alternativas. Educare, 20(66), 237-247. https://www.redalyc.org/pdf/356/35649692005.pdf

Beluche, O. (2016). La pedagogía del opresor: educación por competencias. Otras Voces en Educación, (1). http://otrasvoceseneducacion.org/archivos/10566

Betallán, G. (2003). El poder y la autoridad en la escuela. La conflictividad de las relaciones escolares desde la perspectiva de los docentes de infancia. Revista Mexicana de Investigación Educativa, 8(19), 679-704. https://www.redalyc.org/pdf/140/14001906.pdf

Diálo@os 
De Puellez, M. (1993). Estado y educación en las sociedades europeas. Revista lberoamericana de Educación, (1), 35-57. https://doi.org/10.35362/rie103006

Del Pozo, R. (2016). La autogestión sin solidaridad conduce al corporativismo. Segundo Encuentro Euro-mediterráneo "La economía de los trabadorxs". Viome, Tesalónica, Grecia.

https://www.economiasolidaria.org/reas-castilla-y-leon/noticias/la-autogestion-sin-solidaridad-conduce-al-corporativismo

Espinosa, M. (2020). La escuela primaria en el siglo XX. Consolidación de un invento.

http://biblioweb.tic.unam.mx/diccionario/htm/articulos/sec 26.htm

Gutiérrez, D. (2013). Derecho y políticas de libre competencia en América Latina.

https://lalibrecompetencia.com/2013/07/29/el-derecho-a-la-libre-competencia-economica-segun-la-cconstitucional-de-colombia/

Narro, J. (2018). Justicia para los Pueblos Originarios. Un asunto a resolver por el gobierno de la 4a transformación. La jornada. México. https://ljz.mx/2018/09/13/justicia-para-los-pueblos-originarios-un-asunto-a-resolver-por-el-gobierno-de-la-4a-transformacion/

Robles, L. (2011). Nuevo modelo educativo: sin reprobados; que todos aprendan. Excélsior. México. https://www.excelsior.com.mx/2011/06/16/nacional/745256

Taub, E. (2020). Abraham, la imperfección del mundo y el origen de una ética de la responsabilidad. http://www.gemrip.org/abraham-la-imperfeccion-del-mundo-y-el-origen-de-una-etica-de-la-responsabilidad/

Taylor, L. (2014). A contracorriente: navegar el flujo y el reflujo de los afectos sociales en la Educación para la Justicia Global. Rizoma Freireano, (16). http://www.rizoma-freireano. org/a-contracorriente-navegar-el-flujo-y-el-reflujo-de-los-afectos-sociales-en-la-educacion-para-la-justicia-global--lisa-k-taylor-

Zoellick, R. (2007). Una globalización incluyente y sostenible. Texto preparado para la intervención en el Banco Mundial. Washington: The National Press Club. https://www.bancomundial. org/es/news/speech/2007/10/10/an-inclusive-sustainable-globalization

\section{Bibliografía consultada}

Lipovetsky, G. (2010). El imperio de lo efímero. La moda y su destino en las sociedades modernas. Barcelona: Anagrama.

Lipovetsky, G. (2011). El crepúsculo del deber. La ética indolora de los nuevos tiempos democráticos. Barcelona: Anagrama.

Magallón, M. (2009). Modernidad alternativa: viejos retos y nuevos problemas. México: UNAM. 